\title{
Acausality in Gowdy spacetimes
}

\author{
Hernando Quevedd* \\ Instituto de Ciencias Nucleares \\ Universidad Nacional Autónoma de México \\ A.P. 70-543, México D.F. 04510, México \\ and \\ Department of Physics \\ University of California \\ Davis, CA 95616
}

(Dated: December 10, 2018)

\begin{abstract}
We present a parametrization of $T^{3}$ and $S^{1} \times S^{2}$ Gowdy cosmological models which allows us to study both types of topologies simultaneously. We show that there exists a coordinate system in which the general solution of the linear polarized special case (with both topologies) has exactly the same functional dependence. This unified parametrization is used to investigate the existence of Cauchy horizons at the cosmological singularities, leading to a violation of the strong cosmic censorship conjecture. Our results indicate that the only acausal spacetimes are described by the Kantowski-Sachs and the Kerr-Gowdy metrics.
\end{abstract}

*Electronic address: quevedo@nuclecu.unam.mx 


\section{INTRODUCTION}

Gowdy cosmologies 1] have been studied for more than 30 years, especially motivated by the desire to understand the mathematical and physical structure of singularities in cosmological spacetimes (globally hyperbolic pseudo-Riemannian manifolds with compact Cauchy spatial hypersurfaces which satisfy Einstein's field equations). It has been long suggested [2] that a singularity is characterized either by a blow up of the curvature and tidal forces, or by a breakdown of causality. However, it is not clear when either possibility is to be expected. The singularity theorems [3] state that reasonable matter evolves from regular data into a singularity, when the evolution is governed by Einstein's equations. The singularities that form in such a process are characterized by causal geodesic incompleteness. The generic nature of these singularities, however, is not described by the singularity theorems. In particular, the question about the blow up of the curvature and tidal forces at the singularity and about the existence of a horizon that "hides" the singularity cannot be addressed with the methods used to study geodesic incompleteness.

Cosmic censorship conjectures state that a singularity must be hidden by an event horizon (weak conjecture) or not be detectable by timelike observers until they fall into it (strong conjecture). In the context of the initial value problem of general relativity, the strong cosmic censorship (SCC) asserts that in the space of allowed initial data there exists only a very small set which evolves into spacetimes that can be extended beyond their maximal domain of dependence into acausal regions. Such extendible spacetimes are characterized by the existence of Cauchy horizons. When a Cauchy horizon exists in a given spacetime, one expects that, in principle, timelike geodesics can be found which become closed after crossing the Cauchy horizon. This would indicate a violation of the SCC conjecture.

In the context of Gowdy cosmological models, the fundamental questions concerning global existence of solutions [4] and the existence of cosmological singularities [5] were analyzed in detail for the case of a $T^{3}$ topology, whereas the $S^{1} \times S^{2}$ case has received less attention. In general, it has been shown that these spacetimes possess cosmological singularities, and the asymptotic behavior of the metric and curvature near these singularities has been the subject of numerous studies (for a recent review, see [6]). The question of the curvature behavior at the cosmological singularity has recently been answered in quite

general terms in 7] and [8] for the case of a toroidal topology. According to Isenberg and 
Moncrief [5], a Gowdy model is called generic when all the corresponding curvature invariants blow up at the cosmological singularity for all values of the spatial coordinate. If the curvature invariants happen to remain regular at the cosmological singularity, the spacetime is called non-generic. In this case, the singularity could become a Cauchy horizon and, in principle, it should be possible to extend the spacetime across the Cauchy horizon to include non-globally hyperbolic acausal regions, indicating a violation of the SCC conjecture. Consequently, if we want to maintain the predictability of Einstein's equations, we should avoid the existence of Cauchy horizons. It follows that within the class of Gowdy models, the SCC conjecture can be violated only in non-generic models. This paper is concerned with the search for non-generic models with $T^{3}$ and $S^{1} \times S^{2}$ topologies. We will show that only a very small set (the Kerr-Gowdy metric) of Gowdy models are non-generic so that the SCC conjecture holds in most models.

This paper is organized as follows. In Section II we present a particular parametrization and a system of coordinates in which the field equations exhibit the same functional dependence for $T^{3}$ and $S^{1} \times S^{2}$ models. This allows us to derive the general solution for Gowdy

polarized models in Section [II. In Section [V] we investigate the question of existence of Cauchy horizons in the general solution. Finally, in Section $\nabla$ we summarize our results.

\section{A UNIFIED PARAMETRIZATION OF GOWDY COSMOLOGIES}

Gowdy cosmological models are inhomogeneous time-dependent solutions of Einstein's vacuum equations. The most general topology of the spatial hypersurfaces can be shown to be either $T^{3}$ or $S^{1} \times S^{2}$. In most studies only the special case of polarized $T^{3}$ models has been considered. Here we will use a particular parametrization of the corresponding line element which allows us to analyze both cases in quite general terms. Let us introduce the line element

$$
d s^{2}=e^{-\lambda / 2+\tau / 2}\left(-e^{-2 \tau} d \tau^{2}+d \chi^{2}\right)+\sqrt{g_{2}}\left[e^{P}(d \sigma+Q d \delta)^{2}+e^{-P} d \delta^{2}\right],
$$

where $P, Q$, and $\lambda$ depend on the non-ignorable coordinates $\tau$ and $\chi$. The cosmological models are compactified by requiring that $0 \leq \chi, \sigma, \delta \leq 2 \pi$. The function $g_{2}$ corresponds to the determinant of a two-metric and satisfies the differential equation $\left(t=e^{-\tau}\right)$

$$
\frac{g_{2, t t}}{g_{2}}-\frac{1}{2}\left(\frac{g_{2, t}}{g_{2}}\right)^{2}-\frac{g_{2, \chi \chi}}{g_{2}}+\frac{1}{2}\left(\frac{g_{2, \chi}}{g_{2}}\right)^{2}=0
$$


which follows from the vacuum field equations. The special case of a $T^{3}$ topology is obtained from the solution

$$
g_{2}=t^{2}=e^{-2 \tau}
$$

while the $S^{1} \times S^{2}$ case corresponds to

$$
g_{2}=c^{2} \sin ^{2} e^{-\tau} \sin ^{2} \chi
$$

where $c$ is a real constant. It turns out that the field equations reduce to a set of two second-order coupled partial differential equations for $P$ and $Q$ and a set of two first-order partial differential equations for $\lambda$ which can be integrated by quadratures once $P$ and $Q$ are known. In the following analysis we will consider only the main field equations for $P$ and $Q$. To handle these equations it is appropriate to consider the corresponding Einstein-Hilbert Lagrangian $\mathcal{L}=\sqrt{-g} R$ which after a Legendre transformation can be written as [9]

$$
\mathcal{L}=\frac{1}{2} e^{\tau} \sqrt{g_{2}}\left[P_{, \tau}^{2}-e^{-2 \tau} P_{, \chi}^{2}+e^{2 P}\left(Q_{, \tau}^{2}-e^{-2 \tau} Q_{, \chi}^{2}\right)\right]
$$

This corresponds also to the Lagrangian of a non-linear sigma model $S L(2, R) / S O(2)$ where the target space turns out to be a hyperbolic space with metric $d s_{2}^{2}=d P^{2}+e^{2 P} d Q^{2}$, when $Q$ and $P$ are used as coordinates of the target space [1]. Accordingly, the Gowdy cosmologies can be considered in general as a special case of the non-linear sigma model $S L(2, R) / S O(2)$. The variation of the Lagrangian (15) yields

$$
\begin{gathered}
P_{, \tau \tau}-e^{-2 \tau} P_{, \chi \chi}+P_{, \tau}\left(1+g_{2}^{-1} g_{2, \tau}\right)-e^{-2 \tau} g_{2}^{-1} g_{2, \chi} P_{, \chi}-e^{-2 P}\left(Q_{, \tau}^{2}-e^{-2 \tau} Q_{, \chi}^{2}\right)=0, \\
Q_{, \tau \tau}-e^{-2 \tau} Q_{, \chi \chi}+Q_{\tau}\left(1+g_{2}^{-1} g_{2, \tau}\right)-e^{-2 \tau} g_{2}^{-1} g_{2, \chi} Q_{, \chi}+2\left(P_{, \tau} Q_{, \tau}-e^{-2 \tau} P_{, \chi} Q_{, \chi}\right)=0 .
\end{gathered}
$$

The important aspect about the Lagrangian (15) is that it can be used to derive a more compact representation of the main field equations. This is the so called Ernst representation [10] which was originally derived for axisymmetric stationary spacetimes and has been generalized to include different types of spacetimes with two Killing vector fields [11]. In the case of Gowdy cosmologies (11), the Ernst equation can be written as 12]

$$
\left(1-\xi \xi^{*}\right)\left[\nabla^{2} \xi+\frac{1}{2} \nabla \ln \left(g_{2}\right) \nabla \xi\right]+2 \xi^{*}(\nabla \xi)^{2}=0
$$

where $\nabla=\left(\partial_{t}, i \partial_{\chi}\right)$ is a complex vector operator, $t=e^{-\tau}$, and the Ernst potential is defined as

$$
\xi=\frac{1-\sqrt{g_{2}} e^{P}-i R}{1+\sqrt{g_{2}} e^{P}+i R}, \quad R_{, t}=\sqrt{g_{2}} e^{2 P} Q_{, \chi}, \quad R_{, \chi}=\sqrt{g_{2}} e^{2 P} Q_{, t}
$$


Here an asterisk represents complex conjugation. By using Eq.(2), it is straightforward to show that in fact the Ernst equation (8) is equivalent to the main field equations (6) and (17). As we can see from the above expressions, in this parametrization the only difference between $T^{3}$ and $S^{1} \times S^{2}$ models lies in the determinant $g_{2}$. This difference can be "hidden" if we success in finding a representation in which the determinant coincides for both cases. This can easily be achieved by introducing coordinates $x$ and $y$ for the $T^{3}$ case as

$$
e^{-2 \tau}=c^{2}\left(1-x^{2}\right)\left(1-y^{2}\right), \quad \chi=c x y
$$

with $x^{2} \leq 1$ and $y^{2} \leq 1$, and the coordinates $\tilde{x}$ and $\tilde{y}$ for the $S^{1} \times S^{2}$ topology as

$$
\tilde{x}=\cos e^{-\tau}, \quad \tilde{y}=\cos \chi,
$$

so that the determinant becomes $g_{2}=c^{2}\left(1-x^{2}\right)\left(1-y^{2}\right)$ for the $T^{3}$ case, while for the $S^{1} \times S^{2}$ case we get the same expression with $x$ and $y$ replaced by $\tilde{x}$ and $\tilde{y}$, respectively. In these coordinates, the Ernst equation (8) can be written as

$$
\left(1-\xi \xi^{*}\right)\left\{\left[\left(1-x_{*}^{2}\right) \xi_{, x_{*}}\right]_{, x_{*}}-\left[\left(1-y_{*}^{2}\right) \xi_{, y_{*}}\right]_{, y_{*}}\right\}+2 \xi^{*}\left[\left(1-x_{*}^{2}\right) \xi_{, x_{*}}^{2}-\left(1-y_{*}^{2}\right) \xi_{, y_{*}}^{2}\right]=0
$$

where $x_{*}=x, y_{*}=y$ for $T^{3}$ models, and $x_{*}=\tilde{x}, y_{*}=\tilde{y}$ for $S^{1} \times S^{2}$ models. Thus, we have

obtained a representation in which the main field equations for all Gowdy cosmologies have the same functional dependence. For the sake of completeness, we also present the final form of the general line element in the new coordinates:

$$
d s^{2}=e^{-\lambda_{*} / 2}\left(-\frac{d x_{*}^{2}}{1-x_{*}^{2}}+\frac{d y_{*}^{2}}{1-y_{*}^{2}}\right)+c\left(1-x_{*}^{2}\right)^{1 / 2}\left(1-y_{*}^{2}\right)^{1 / 2}\left[e^{P}(d \sigma+Q d \delta)^{2}+e^{-P} d \delta^{2}\right],
$$

where

$$
e^{-\lambda_{*} / 2}=c^{3 / 2} \frac{x^{2}-y^{2}}{\left(1-x^{2}\right)^{1 / 4}\left(1-y^{2}\right)^{1 / 4}} e^{-\lambda / 2}, \quad e^{-\lambda_{*} / 2}=(\arccos \tilde{x})^{-1 / 2} e^{-\lambda / 2},
$$

for the $T^{3}$ and $S^{1} \times S^{2}$ models, respectively. In this parametrization, the only functional difference between both topologies is contained in the form of the metric function $\lambda_{*}$.

\section{THE GENERAL SOLUTION}

The importance of the parametrization of the last section is that it allows us to investigate both types of Gowdy models with the same functional dependence. Let us consider now the 
special polarized case, $Q=0$. From the definition of the Ernst potential (9) we see that the function $R$ reduces to a constant which, without loss of generality, can be put as $R=0$. A straightforward calculation shows that in this case the Ernst equation (8) reduces to

$$
\left[\left(1-x_{*}^{2}\right) P_{, x_{*}}\right]_{, x_{*}}-\left[\left(1-y_{*}^{2}\right) P_{, y_{*}}\right]_{, y_{*}}=0
$$

an equation which can be solved by separation of variables and whose general solution can be written as an infinite series [13]

$$
P=\sum_{\nu}\left[a_{\nu} P_{\nu}\left(x_{*}\right)+b_{\nu} Q_{\nu}\left(x_{*}\right)\right]\left[c_{\nu} P_{\nu}\left(y_{*}\right)+d_{\nu} Q_{\nu}\left(y_{*}\right)\right]
$$

where $\nu$ is a constant, $P_{\nu}$ and $Q_{\nu}$ are the Legendre functions of first and second kind, respectively, and $a_{\nu}, b_{\nu}, c_{\nu}$ and $d_{\nu}$ are real constants.

It is now a question of analyzing the behavior of the functions $P_{\nu}$ and $Q_{\nu}$ within the interval $-1 \leq x_{*}, y_{*} \leq+1$ to determine which of the solutions contained in (16) are physical relevant. For instance, one should impose that the function $P$ is periodic in the angular coordinate $\chi$. This condition is identically satisfied in the $S^{1} \times S^{2}$ case because the angular dependence of the general solution is determined through $y_{*}=\tilde{y}=\cos \chi$. In the $T^{3}$ case one can also show [13] that (16) contains an infinite number of periodic solutions. Furthermore, it is possible to analyze the asymptotic behavior of the solution in quite general terms. If $\nu$ is not an integer, the function $P_{\nu}$ diverges at $x_{*}=-1$. But if $\nu$ is an integer number, say $n$, then $P_{\nu}$ becomes the Legendre polynomials $P_{n}$ which are free of singularities for any values in the interval $-1 \leq x_{*} \leq+1$. On the other hand, the function $Q_{\nu}$ possesses singularities at $x_{*}= \pm 1$ for all integer and non-integer values of $\nu$.

An additional important aspect of the solution presented above is that it coincides exactly with the general static axisymmetric solution of Einstein's vacuum equations [14] in prolate spheroidal coordinates. Moreover, the Ernst equation (8) is functionally equivalent to the main field equations of stationary axisymmetric spacetimes. This functional equality is due to the fact that both Gowdy cosmologies and stationary axisymmetric spacetimes possess a set of two commuting Killing vector fields. The Ernst equation has been used to analyze the internal symmetries of the field equations and to develop the modern solution generating techniques. In particular, in a recent work [15] it was shown that all the Gowdy $T^{3}$ cosmologies can be generated from the data at the initial singularity. The results presented here suggest that a similar procedure can be developed for $S^{1} \times S^{2}$ models. Our results also 
explain why the Kantowski-Sachs metric (the region inside the horizon of the Schwarzschild metric) and the Kerr-Gowdy metric (the region inside the horizons of the Kerr metric) can be interpreted both as $T^{3}[15]$ and $S^{1} \times S^{2}$ Gowdy cosmological models [12].

\section{CAUCHY HORIZONS}

In the previous section we have derived a unified parametrization for all types of Gowdy cosmologies and found the general polarized solution. In this section we will show that this general solution contains all the information necessary to determine which spacetimes can allow the existence of Cauchy horizons. As we mentioned in the Introduction, a cosmological singularity can become a Cauchy horizon if the curvature is regular at the singularity.

The cosmological singularities of $S^{1} \times S^{2}$ Gowdy models in the original parametrization $(\tau, \chi)$ correspond to the limits $\tau \rightarrow \infty$ and $\tau \rightarrow-\ln \pi$. In the coordinates $\tilde{x}, \tilde{y}$ described above, this corresponds to the hypersurfaces $\tilde{x} \rightarrow 1$ and $\tilde{x} \rightarrow-1$, respectively. In the case of $T^{3}$ models, the singularity is situated at $\tau \rightarrow \infty$, a limit that in coordinates $x$ and $y$ corresponds to $x^{2} \rightarrow 1$ or $y^{2} \rightarrow 1$. According to the explicit form of the general line element (13), the latter case corresponds to a spatial limit which is not of interest for the study of cosmological singularities (temporal limit). Therefore we can eliminate all possible singularities at $y_{*}^{2}=1$ from the general solution (16). To avoid the singularity of the function $P_{\nu}\left(y_{*}\right)$ at $y_{*}=-1$, we consider only positive integer values of the constant $\nu$, i. e., $\nu=n=0,1,2, \ldots$ Furthermore, the singularities of the function $Q_{n}\left(y_{*}\right)$ at $y_{*}= \pm 1$ can be eliminated by choosing $d_{n}=0$ in (16). Then the general solution reduces to

$$
P=\sum_{n}\left[a_{n} P_{n}\left(x_{*}\right)+b_{n} Q_{n}\left(x_{*}\right)\right] c_{n} P_{n}\left(y_{*}\right) .
$$

We now consider the singularity at $x_{*}= \pm 1$. The Legendre polynomials $P_{n}\left(x_{*}\right)$ and their derivatives have constant regular values at the limits $x_{*}= \pm 1$. So they essentially do not contribute to the behavior of the solution at the cosmological singularity, and we can completely ignore its contribution by choosing $a_{n}=0$. Consequently, the general solution which is of importance at the singularity can be written as

$$
P=\sum_{n} b_{n} Q_{n}\left(x_{*}\right) P_{n}\left(y_{*}\right),
$$

where we have chosen $c_{n}=1$, without loss of generality. The main point now is that the functional dependence of the cosmological solution (18) coincides exactly with the functional 
dependence of static axisymmetric asymptotically flat solutions. The only difference lies in the physical meaning of the coordinates $x_{*}$ and $y_{*}$. While for static solutions both coordinates are spacelike, in polarized Gowdy cosmologies the coordinate $x_{*}$ becomes timelike. The condition of asymptotic flatness, which is used to obtain the general solution for static spacetimes in the form (18), corresponds in polarized Gowdy spacetimes to the condition of considering only those solutions which are non-ignorable for the analysis of the asymptotic behavior near the cosmological singularities. Moreover, the behavior of the general solution (18) near the cosmological singularity corresponds to the near horizon limit of static solutions $x_{*} \rightarrow 1$ (in spherical coordinates, this corresponds to the limit $r \rightarrow 2 m$, where $m$ is the Schwarzschild mass).

Let us now consider the unpolarized case, $Q \neq 0$. The static counterpart of the general polarized solution (18) has been used to derive the most general stationary axisymmetric asymptotically flat spacetime by using solution generating techniques [17]. This generalized solution contains, in particular, the Kerr metric which is the most general (vacuum) black hole solution. In fact, the uniqueness theorems [18] state that the Kerr spacetime is the most general solution with regular horizons. On the other hand, the inner and outer horizon limits of the Kerr metric correspond to $x_{*} \rightarrow-1,+1$, respectively [12]. From the above considerations, it is clear that the general polarized $(Q=0)$ solution (18) can be used to generate the most general unpolarized $(Q \neq 0)$ Gowdy solution which should be considered for analyzing the behavior near the cosmological singularities. In particular, the Kerr-Gowdy solution must be contained as a special case. For this solution, it has been shown [12] that the cosmological singularities are situated at $x_{*} \rightarrow \pm 1$, a limit that coincides with the near horizon limit of the Kerr metric. Using the functional analogy between stationary spacetimes and unpolarized Gowdy cosmologies and the black hole uniqueness theorems, we can conclude that the Kerr-Gowdy metric is the most general solution with a regular curvature behavior near the cosmological singularities, i.e. it is the most general non-generic Gowdy spacetime. The hypersurfaces $x_{*}= \pm 1$ could become Cauchy horizons so that the Kerr-Gowdy spacetime could be extended to include acausality regions where the SCC conjecture is violated. In fact, in a recent work [19] several generalizations of the KantowskiSachs and the Kerr-Gowdy spacetimes have been analyzed, finding in all of them a curvature blow up at the cosmological singularities which does not allow the formation of Cauchy horizons. 


\section{CONCLUSIONS}

In this work we have found a unified parametrization for $T^{3}$ and $S^{1} \times S^{2}$ Gowdy cosmological models. This unified parametrization allowed us to find the general polarized solution for both types of Gowdy models in terms of Legendre functions of first and second kind. We analyzed the general solution which determines the behavior near the cosmological singularities. Using the functional analogy between Gowdy cosmologies and stationary axisymmetric spacetimes, we concluded that the Kerr-Gowdy metric is the most general Gowdy cosmological model in which acausality regions might exist that violate the SCC conjecture. This result agrees with the conclusion of [5] that only a very small set of polarized Gowdy spacetimes could be extended into an acausal region, across a Cauchy horizon. In fact, we have shown that this set includes only the Kantowski-Sachs spacetime. For unpolarized models, our results show that only the Kerr-Gowdy metric could possess Cauchy horizons. It would be interesting to show explicitly the existence of closed timelike curves beyond the Cauchy horizons of the Kerr-Gowdy metric. In the special polarized case of the

Kantowski-Sachs metric a preliminary study [20] seems to indicate that this is impossible because for all timelike geodesics there exists a focusing point which does not allow them to cross the horizon. A more detailed analytical study is necessary in order to completely clarify this question.

\section{ACKNOWLEDGMENTS}

In 1999, Mike Ryan presented a seminar at UNAM about the problem of finding exact solutions which would describe the gravitational field in Gowdy cosmologies. This was the starting point of a joint collaboration, together with Octavio Obregón, in which we are exploring several methods of generating exact Gowdy cosmologies and are, simultaneously, reinterpreting stationary spacetimes as Gowdy cosmologies. I would like to thank Mike for drawing me closer to the world of the initial singularity. It is a great pleasure to dedicate this work to my friend and colleague Mike Ryan on the occasion of his sixtieth birthday.

This work was supported by US DOE grant DE-FG03-91ER 40674, DGAPA-UNAM grant IN112401, CONACyT-Mexico grant 36581-E, and UC MEXUS-CONACyT (Sabbati- 
cal Fellowship Program).

[1] R. Gowdy, Phys. Rev. Lett. 27, 826 (1971); Ann. Phys. (N.Y.) 83, 203 (1974).

[2] C. J. S. Clarke, J. Math. Anal. Appl. 88, 270 (1982).

[3] S. W. Hawking and G. F. R. Ellis, The large scale structure of spacetime (Cambridge University Press, Cambridge, UK, 1973).

[4] V. Moncrief, Ann. Phys., NY 132, 87 (1981).

[5] J. Isenberg and V. Moncrief, Ann. Phys. (N.Y.) 199, 84 (1990).

[6] B. Berger, Liv. Rev. Rel. 5, 1 (2002).

[7] P. T. Chruściel and K. Lake, Class. Quantum Grav. 21, S153 (2004).

[8] H. Ringström, Class. Quantum Grav. 21, S305 (2004).

[9] J. Cortez, D. Núñez, and H. Quevedo, Int. J. Theor. Phys. 40, 251 (2001).

[10] E. J. Ernst, Phys.Rev. D 168, 1415 (1968).

[11] J. B. Griffiths, Colliding plane waves in general relativity (Oxford University Press, Oxford, 1991); N. Breton, A. Feinstein and J. Ibañez, Class. Quantum Grav. 9, 2437 (1992); N. Breton, Gen. Rel. Grav. 25, 567 (1993).

[12] O. Obregon, H. Quevedo, and M. P. Ryan, Phys. Rev. D 65, 024022 (2001).

[13] A. Erdelyi, W. Magnus, F. Oberhettinger, and F. G. Tricomi, Higher transcendental functions (University of Chicago Press, Chicago, 1953).

[14] G. Erez and N. Rosen, Bull. Res. Council of Israel 8F, 47 (1959); C. Reina and A. Treves, Gen. Rel. Grav. 7, 817 (1976).

[15] A. Sanchez, A. Macias, and H. Quevedo, J. Math. Phys. 45, 1849 (2004).

[16] H. Stephani, D. Kramer, M. MacCallum, C. Hoenselaers, and E. Herlt, Exact solutions of Einstein's field equations (Cambridge University Press, Cambridge UK, 2003).

[17] H. Quevedo, Phys. Rev. D 33, 324 (1986); Fortschr. Phys. 38, 733 (1990).

[18] M. Heusler, Liv. Rev. Rel. 1, 6 (1998).

[19] O. Obregon, H. Quevedo, and M. P. Ryan, Time and "angular" dependent backgrounds from stationary axisymmetric solutions, Phys. Rev. D 68, (2004) in press, gr-qc/0404003.

[20] H. Quevedo and M. Ryan, in Mathematical and Quantum Aspects of Relativity and Cosmology, edited by S. Cotsakis and G.W. Gibbons (Springer-Verlag, Berlin, 2000), gr-qc/0305001. 